\title{
Expression and Clinical Significance of Ki67 in Common Gastrointestinal Tumours
}

\author{
Fangyu Shi, Yunnan Dai, Yiying Bai, Qingshan Li $^{*}$ \\ Affiliated Hospital of Chengde Medical University, Chengde, China \\ Email: 757364123@qq.com, 459851575@qq.com, 1085924089@qq.com, *libing200865@126.com
}

How to cite this paper: Shi, F.Y., Dai, Y.N., Bai, Y.Y. and Li, Q.S. (2021) Expression and Clinical Significance of Ki67 in Common Gastrointestinal Tumours. Open Journal of Gastroenterology, 11, 295-306. https://doi.org/10.4236/ojgas.2021.1112029

Received: November 16, 2021

Accepted: December 28, 2021

Published: December 31, 2021

Copyright ( 2021 by author(s) and Scientific Research Publishing Inc. This work is licensed under the Creative Commons Attribution International License (CC BY 4.0).

http://creativecommons.org/licenses/by/4.0/

\begin{abstract}
Ki67 is a marker of cell proliferation that is expressed in the S, G2 and M phases but not in the G0 phase of the cell cycle. Several recent studies showed that the expression of Ki67 is closely related to the occurrence and development of gastrointestinal tumours. The Ki67 index is closely related to the degree of differentiation, invasion, metastasis and prognosis of gastrointestinal tumours. This review describes the relationship between the Ki67 index and degree of malignancy, therapeutic effect and prognosis of gastrointestinal tumours.
\end{abstract}

\section{Keywords}

Gastrointestinal Neoplasms, Ki67, Pathological Features, Prognosis

\section{Introduction}

Despite the recent progress in the diagnosis and treatment of gastrointestinal tumours, the rate of incidence and mortality has not decreased significantly. Therefore, effective diagnostic and therapeutic targets need to be urgently identified. Screening for specific indicators guiding individualized treatment has become the focus of studies on gastrointestinal tumours. Results of previous studies have shown that Ki67 is a nuclear antigen closely related to cell proliferation and can be used as an important marker to evaluate cell proliferation. Therefore, Ki67 is widely used in the research and clinical application of malignant tumours. Recent studies showed that Ki67 can be used as a clinically valuable biomolecule to predict the prognosis of tumours of the digestive system, such as gastric cancer, colorectal cancer, liver cancer and oesophageal cancer; moreover, Ki67 can be used as an index to determine the therapeutic effect to a certain extent. In this review, we discussed recent studies on the correlation between Ki67 and common gastrointestinal tumours to clarify its value as a tumour cell proli- 
feration and prognostic marker.

\section{Molecular Biological Characteristics of Ki67}

\subsection{Structure and Function of Ki67}

Ki67 was first discovered by Gerdes J et al. [1] in the 1980s. Ki67 was identified as a nuclear antigen in Hodgkin's lymphoma. Ki67 is a non-histone nuclear protein. The gene encoding Ki67 is located on chromosome 10, transcribed and processed by 15 exons, and encodes nuclear macromolecular proteins with relative molecular weights of 345,000 and 395,000 [2]. The half-life of Ki67 protein is $1-1.5 \mathrm{~h}$. The expression of Ki67 is precisely regulated during the cell cycle. Ki67 is not expressed in the G0 phase and early G1 phase; however, the expression level gradually increases in the G1, G2 and S phases and reaches a peak in the $\mathrm{M}$ phase; subsequently, the Ki67 expression decreases sharply in the later and late stage of mitosis [3]. The expression level of Ki67 is regulated by a dynamic balance between synthesis and degradation. The expression of Ki67 protein is regulated by the process of cell mitosis, and Ki67 undergoes phosphorylation and dephosphorylation during mitosis. Therefore, Ki67 is vulnerable to degradation by proteases [4]. Sang D et al. [5] showed that Ki67 binds to DNA, RNA and other proteins in a complex manner and thus, it may play an important role in cell division. These characteristics make Ki67 a good cell proliferation marker. The expression level of Ki67 increases significantly during tumour proliferation.

\subsection{Clinical Method for Detection of Ki67 and Definition of Positive Rate}

Currently, two clinical methods are available to detect Ki67 expression; one is a semi-quantitative analysis of MKI67 messenger RNA extracted from frozen or formalin-fixed paraffin embedded tissues, and the other is an immunohistochemical method for detecting Ki67 based on cell staining ratio [6]. Immunohistochemical analysis is the clinically most widely used method. Clinically, nuclear staining is observed microscopically under a $40 \times$ magnification to calculate the Ki67 index. Most pathology departments in China use manual counting as the main method to evaluate the Ki67 index; however, this is a subjective method, and the pathologists involved in the counting have a varied level of expertise. Therefore, a clinically more accurate and efficient method needs to be developed. To date, a consensus has not been achieved about the clinically optimal high and low levels of Ki67 expression. It is reported that the maximal difference between optimal high and low levels of Ki67 expression is more than 30\% [7]. Therefore, determination of a more reasonable optimal level of Ki67 expression is a challenge that needs to be addressed.

\subsection{Ki67 and Tumour Prognosis}

In the recent years, many studies have shown that Ki67 is an important marker of tumour prognosis. Ki67 is positively correlated with the degree of malignancy 
of the tumour. Additionally, Ki67 is an important marker for determining the nature and invasiveness of the tumour and for predicting the tumour prognosis. Qian Xia et al. [8] showed that in patients with nasal natural killer (NK)/T-cell lymphoma, the effect of radiotherapy was lower in Ki67-positive patients than in Ki67-negative patients. Ki67 can be used as an evaluation index for the prognosis of nasal NK/T-cell lymphoma. A study by He $\mathrm{H}$ et al. [9] on non-small-cell lung cancer revealed that Ki67 is related to pathological factors such as pathological classification and tumour differentiation. The 2-year survival rate and median survival duration of Ki67-positive patients was lower than that of Ki67-negative patients. A meta-analysis by Zhang Y [10] and Yan et al. revealed that patients with breast cancer with high expression levels of Ki67 had a significantly higher disease-free survival than those with low expression levels of Ki67. Zheng C et al. [11] showed that Ki67 is related to the FIGO stage and lymph node metastasis in ovarian cancer. The median survival duration of patients with ovarian cancer with serum levels of Ki67 $\geq 50 \mathrm{pg} / \mathrm{mL}$ was shorter than that of patients with ovarian cancer with serum Ki67 levels $<50 \mathrm{pg} / \mathrm{mL}$. In a study on patients with cervical cancer by Cao Q et al. [12], a statistically significant difference was observed between the Ki67 expression levels and the degree of differentiation, clinical stages and lymph node metastasis. The 3-year survival rate of Ki67-positive patients was lower than that of Ki67-negative patients. Huang T et al. [13] examined the role of Ki67 as a risk factor affecting the prognosis of prostate cancer. A high Ki67 index indicates a high risk of death and hence a worse prognosis. Results of a retrospective study in various cancers have shown that Ki67 has been widely used as a potential proliferation marker.

\section{Relationship between Ki67 and Gastrointestinal Tumours}

\subsection{Ki67 and Gastric Cancer}

Gastric cancer is the fifth most common cancer and the third cause of cancer-related death worldwide [14]. Gastric cancer is associated with a relatively poor prognosis and thus poses a serious threat to human health. More than $70 \%$ of the new cases of gastric cancer occur in developing countries, and the cases in eastern Asia account for about $50 \%$ of those reported worldwide, and they are mainly concentrated in China [15]. China is a country with a high incidence of gastric cancer and serious disease burden. Therefore, prevention and treatment of gastric cancer are of prime importance. To date, reasonable and effective molecular markers to accurately reflect the prognosis of patients with gastric cancer have not been identified. Pathologists in China agree that the expression level of Ki67 can be used to evaluate the proliferation level of gastric cancer cells and provide a significant reference for the evaluation of the efficacy of postoperative chemotherapy [16].

Previous studies [17] have identified the known pathological factors that can affect the postoperative prognosis of patients with gastric cancer, including TNM stage, presence of lymph node metastasis, the size of the tumour tissue, the 
degree of tumour invasion, presence of nerve invasion and presence of blood vessel invasion. To date, a consensus on whether a correlation exists between Ki67 and the above clinicopathological parameters affecting prognosis has not been established. Previous studies [18] [19] have shown that Ki67 is related to TNM stage, lymph node metastasis and degree of tumour invasion in gastric cancer, that is, specimens obtained from patients with late stage gastric cancer, lymph node metastasis and deep invasion showed high expression levels of Ki67. In addition, some studies showed that the level of Ki67 expression is high in gastric cancer tissues in large tumour tissue [20] and vascular tumour thrombus [21]. Therefore, Ki67 is considered to be related to the occurrence of gastric cancer. To some extent, it reflects the malignant degree and prognosis of patients with gastric cancer. However, some studies have not been able to identify a relationship between Ki67 and pathological factors such as tissue size and TNM stage of gastric cancer [22]. The different conclusions obtained from different studies may be related to the inconsistency in the optimal value of Ki67 and applied statistical methods, which remains to be further confirmed by a large number of studies.

Zhou Y et al. [23] selected 155 samples from patients with gastric cancer (76 patients with liver metastasis of gastric cancer were in the liver metastasis group and 79 patients with non-liver metastasis of gastric cancer were in the non-metastasis group) for study. The chi-square test revealed that the number of Ki67-positive patients was significantly higher in the metastasis group than in the non-metastasis group, suggesting that upregulation of Ki67 may be involved in the occurrence of liver metastasis in gastric cancer Thus, Ki67 may be used to predict liver metastasis of gastric cancer. Chen $\mathrm{T}$ et al. [21] collected 167 specimens from patients with gastric cancer; they performed Kaplan-Meier analysis and showed that patients with low expression levels of Ki67 had a statistically significantly longer 5-year recurrence-free survival time. Furthermore, the results of multivariate Cox regression analysis revealed that the recurrence risk of patients with expression level of Ki67 $<10 \%$ was 0.318 times that of patients with Ki67 expression level $\geq 10 \%$. Therefore, detection of Ki67 may be helpful in predicting the prognosis of patients with gastric cancer and providing a reference for formulating corresponding treatment strategies. When combined with forkhead box P1 (FOXP1), which is a new biomarker, Ki67 may improve the ability of predicting prognosis to a certain extent. Hua Y [24] examined 69 patients with advanced gastric cancer treated with fluorouracil-based combined adjuvant chemotherapy after operation. The progression-free survival duration of Ki67-positive patients was significantly lower than that of Ki67-negative patients, and Ki67-positive patients were more predisposed to disease progression. Moreover, the sensitivity and short-term efficacy of chemotherapy in Ki67-positive patients was higher than that in Ki67-negative patients, suggesting that the Ki67 expression level can provide a reference for the evaluation of efficacy of chemotherapy.

Most studies have shown that the rate of Ki67 positivity is high in gastric can- 
cer specimens with late stage, lymph node metastasis and deep infiltration. The upregulation of Ki67 expression level can predict liver metastasis of gastric cancer to a certain extent. Compared with Ki67-negative patients, Ki67-positive patients have shorter relapse-free survival time, progression-free survival time, high sensitivity to fluorouracil drugs and good short-term curative effect. Therefore, when used in combination with other biomarkers, Ki67 may show better results.

\subsection{Ki67 and Colorectal Cancer}

Colorectal cancer (CRC) has the highest rate of incidence and mortality in China [25]. The current therapeutic approach of CRC is mainly based on surgery and a combination of chemotherapy and radiotherapy. TNM staging developed by the American Joint Committee on cancer is currently one of the most widely used system for staging of CRC, which is of great significance for evaluation of the prognosis and providing guidance for follow-up treatment. However, in clinical practice, patients with the same stage of cancer may have different prognosis [26]. Therefore, the prognosis should be evaluated along with other indicators to formulate an appropriate treatment plan. Ki67 is closely related to the occurrence and development of human CRC and is considered to be an important predictor of CRC [27].

A previous study [28] showed that the overall survival and the 5-year survival rate of patients with CRC with pathological types of low differentiation, vascular tumour thrombus, nerve invasion, lymph node metastasis and TNM stages III IV were significantly lower than those with high differentiation, no vascular tumour thrombus, nerve invasion, lymph node metastasis and TNM stages I - II. Previous studies investigating the relationship between Ki67 and pathological features affecting the prognosis of patients [29] [30] have revealed that Ki67 is related to TNM stage, lymph node metastasis and degree of differentiation of $\mathrm{CRC}$, that is, the later the stage, the more lymph node metastasis and the lower the degree of differentiation, the higher the expression level of Ki67, suggesting that Ki67 may be involved in the invasion and metastasis of CRC. It is related to the malignant degree and clinical stage of CRC, which provides certain guiding significance for clinical treatment and prognosis. Other related studies [31] have shown that the clinical stage and degree of differentiation are independent of Ki67 but are related to small sample size and regional differences.

Tong G [30] examined 1090 patients with CRC, and their results of Cox multivariate regression analysis revealed that the risk of recurrence of CRC in patients with Ki67 expression levels of 25\% - 50\%, 50\% - 75\% and 75\% - 100\% was 2.59, 4.732 and 6.762 times higher, respectively, than that of patients with Ki67 expression levels of $0 \%-25 \%$. CRC patients with high Ki67 expression levels have a higher risk of recurrence. Therefore, Ki67 was proven to be an independent predictor of the prognosis of CRC. Wei X [31] performed a study with 195 patients with CRC. The results of univariate analysis revealed that the overall survival and the risk of death in Ki67-positive patients were 1.610 times that in 
Ki67-negative patients, which indicates that the Ki67 expression level was related to the overall survival. The risk of death in Ki67-positive patients was significantly higher than that in Ki67-negative patients; however, Ki67 was not an independent risk factor for poor prognosis of CRC. Li H performed a study with [28] 311 CRC patients; they performed univariate survival analysis and showed that the overall survival and 5-year survival rate of CRC patients with high levels of Ki67 were significantly lower than those of patients expressing low levels of Ki67. The results of multivariate analysis revealed that patients positive for carcinoembryonic antigen (CEA) + Ki67 and carbohydrate antigen 19-9 (CA19-9) + Ki67 were independent prognostic factors of CRC, suggesting that the combination of CEA, CA199 and Ki67 may yield better results for the evaluation of prognosis of patients with CRC.

Ki67 is related to TNM stage, lymph node metastasis and degree of differentiation of CRC, suggesting that Ki67 is related to the degree of malignancy. The risk of recurrence and death is higher in patients with high expression levels of Ki67 than in those with low levels of Ki67 expression, suggesting that patients with high Ki67 expression should take more active treatment even if other high-risk factors are absent. Using a combination of tumour markers may increase the level of accuracy to evaluate the prognosis of CRC, which is a new field worthy of exploration.

\subsection{Ki67 and Liver Cancer}

According to the latest data of global cancer, the incidence rate of primary liver cancer is $4.7 \%$; liver cancer is the sixth most common cancer worldwide with a mortality rate of $8.3 \%$, which is the third common cause of cancer-related deaths [25]. Primary liver cancer is a malignant tumour that occurs primarily in hepatocytes or the intrahepatic bile duct epithelial cells. More than $90 \%$ of primary liver cancer is hepatocellular carcinoma (HCC). Surgical resection and liver transplantation are the more effective methods for the treatment of liver cancer. The symptoms of primary liver cancer are not evident in the early stages; therefore, patients in the advanced stage do not have the opportunity of undergoing surgery. Therefore, identification of effective molecular markers and key biological therapeutic targets of HCC is of great significance to improve the outcomes of patients with hepatocellular carcinoma.

Zhao W et al. [32] collected 353 paraffin-embedded specimens of primary liver cancer. The results of univariate analysis revealed that the prognosis of patients with alpha-fetoprotein (AFP) levels $>400 \mu \mathrm{g} / \mathrm{L}$, tumour size $>5 \mathrm{~cm}$ and TNM stage III a was worse than that of patients with AFP levels $\leq 400 \mu \mathrm{g} / \mathrm{L}$, tumour size $\leq 5 \mathrm{~cm}$ and TNM stage I/II, and these results were consistent with those reported by Chen W [33], in that, Ki67 was related to tumour size and TNM stage of liver cancer. The size of the tumour and the stage of liver cancer showed a significant positive correlation with Ki67 expression, indicating that a high level of Ki67 expression is related to poor pathological characteristics and invasive behaviour. The conclusions drawn in a study of Zhang X et al. [34] are 
completely contrary to the abovementioned results, except for the differences caused by the small number of samples and different statistical methods, indicating that large sample size should be used for further investigation.

In a study by Zhao $\mathrm{W}$ et al. [32], the Kaplan-Meier curve revealed a significant difference in the prognosis after hepatectomy between patients with high and low Ki67 expression levels, that is, the cumulative survival function of Ki67-negative patients was significantly higher than that of Ki67-positive patients. Cox univariate and multivariate analyses revealed that the prognosis of patients was significantly correlated with the high expression levels of Ki67 (the death risk of patients with high expression level of Ki67 was 2.709 times and 1.536 times that of patients with low expression level of Ki67), that is, the overall survival time of Ki67-negative patients was higher than that of Ki67-positive patients, which was an independent risk factor for the prognosis of liver cancer. These results are consistent with those of a study by Guo F [35]. Combined detection of CD147 and Ki67 may have more predictive value for the prognosis of liver cancer, and more active comprehensive treatment should be administered to patients with high expression of CD147 and Ki 67. Jia L et al. [36] reported that the expression of Ki67 in the recurrence group was different from that in the cure group, and the expression of Ki67 in the recurrence group was significantly higher than that in the cure group, suggesting that the high expression of Ki67 can predict the risk of recurrence of postoperative liver cancer; the results of this study were similar to those of a study by Zhang X et al. [34].

The larger the tumour is and the later the stage of liver cancer is, the higher the positive rate of Ki67 expression is, suggesting that Ki67-positive patients with liver cancer have a poor prognosis. The risk of recurrence and death of liver cancer patients with high Ki67 expression in tumour tissues was higher than that of those with low Ki67 expression. Combined detection with CD147 or other markers may have more predictive value for the prognosis of liver cancer.

\subsection{Ki67 and Oesophageal Cancer}

Regional differences are present in the incidence of oesophageal cancer in China; moreover, oesophageal cancer is associated with a high mortality rate. The incidence of oesophageal cancer has declined in the recent years, but mortality rate has been the 4 [37]. According to the latest epidemiological survey, the rate of incidence and mortality of oesophageal cancer in China is higher than that in western countries [38]. Although considerable progress has been made in the diagnosis and treatment of oesophageal cancer, it continues to remain one of the malignant tumours with poor prognosis, and the long-term survival rate of patients with oesophageal cancer has not significantly improved. Screening for specific indicators to guide individualised treatment has become a focus of studies on oesophageal cancer.

Wang $\mathrm{H}$ [39] summarised the known pathological factors that can affect the prognosis of patients with oesophageal cancer, such as TNM stage, lymph node 
metastasis and venous invasion. He X [40] considered that a statistically significant difference exists between Ki67 expression and TNM stage and the presence of lymph node metastasis, suggesting that Ki67 is related to poor prognosis.

In a study by Qi X [41], among the 122 newly treated patients with advanced oesophageal cancer, the expression of Ki67 was significantly correlated with the overall survival rate. In addition, Kaplan-Meier survival analysis revealed that there was a significant difference in the postoperative survival curve between patients with high and low expression levels of Ki67. The overall survival of patients with oesophageal squamous cell carcinoma with high expression levels of Ki67 was significantly worse than that of patients with low expression levels of Ki67. The combined detection of Ki67 and vascular endothelial growth factor (VEGF) showed that the postoperative survival curve of patients with high expression levels of Ki67 and VEGF was significantly lower than that of patients without high expression levels of Ki67 and VEGF. Thus, the expression of Ki67 is significantly correlated with the curative effect of radiotherapy, and high Ki67 expression levels indicates a higher degree of tumour cell proliferation of oesophageal squamous cell carcinoma, and thus a lower curative effect of radiotherapy. In a study by Zhao L et al. [42] involving 33 patients with oesophageal basal cell-like squamous cell carcinoma, Kaplan-Meier survival analysis revealed that the cumulative survival rate of patients with low expression levels of Ki67 was significantly higher than that of patients with high expression levels of Ki67. These results were similar to those reported by Qi X et al. [41] A study by Wang $\mathrm{H}$ et al. [39] revealed that no significant difference was observed between the expression levels of Ki67 and overall survival and progression-free survival.

Squamous cell carcinoma accounts for $90 \%$ of all oesophageal cancers; thus, the studies on oesophageal cancer performed thus far mainly focus on squamous cell carcinoma. Previous studies have focussed on oesophageal squamous cell carcinoma. Additionally, studies have been performed on oesophageal adenocarcinoma and oesophageal small cell carcinoma. In a study by Jacobsen F [43] involving 393 patients with oesophageal adenocarcinoma, the results of Cox regression analysis revealed that age and lymph node metastasis were independent prognostic markers, and a statistically significant difference was observed between Ki67 levels and lymph node metastasis. The difference between Ki67 levels and overall survival was statistically significant. The overall survival of patients with high levels of Ki67 expression was shorter than that of patients with low levels of Ki67 expression. In a study of 44 patients with oesophageal small cell carcinoma by Deng HY [44], patients with high Ki67 index ( $>50 \%)$ receiving adjuvant therapy showed a significantly longer survival time than patients without adjuvant therapy. In addition, the results of multivariate analysis revealed that Ki67 index (hazard ratio $[\mathrm{HR}]=0.314,95 \%$ confidence interval $[\mathrm{CI}]: 0.127$ 0.774; $\mathrm{P}=0.012$ ) was an independent prognostic factor in patients with oesophageal small cell carcinoma; therefore, the prognosis of patients with high levels of Ki67 expression was worse than that of patients with low levels of Ki67 expression. 
Ki67 is associated with adverse pathological factors in patients with oesophageal cancer, suggesting that Ki67-positive patients with oesophageal cancer have a poor prognosis. The overall survival of patients with high expression levels of Ki67 in oesophageal cancer was worse than that of patients with low expression levels of Ki67, which was proven in oesophageal squamous cell carcinoma, adenocarcinoma and small cell carcinoma.

\section{Conclusion}

Ki67 plays an important role in the occurrence and development of gastrointestinal tumours and is an important factor for predicting the prognosis of patients with gastrointestinal tumours and designing appropriate treatment strategies. Most relevant studies performed to date are single-centre and retrospective studies, and these limitations may be responsible for the differences in the findings. Multicentre clinical trials and studies with a large sample size are required to demonstrate the validity of relevant research results, and establish routine, accurate, economic and standardised methods for detecting Ki67. In addition, it is necessary to scientifically define the optimal value of Ki67 expression.

\section{Conflicts of Interest}

The authors declare no conflicts of interest regarding the publication of this paper.

\section{References}

[1] Gerdes, J., Schwab, U., Lemke, H., et al. (1983) Production of a Mouse Monoclonal Antibody Reactive with a Human Nuclear Antigen Associated with Cell Proliferation. International Journal of Cancer, 31, 13-20. https://doi.org/10.1002/ijc.2910310104

[2] Gerdes, J., Li, L., Schlueter, C., et al. (1991) Immunobiochemical and Molecular Biologic Characterization of the Cell Proliferation-Associated Nuclear Antigen that Is Defined by Monoclonal Antibody Ki-67. The American Journal of Pathology, 138, 867-873.

[3] Ohashi, R., Namimatsu, S., Sakatani, T., et al. (2018) Prognostic Utility of Atypical Mitoses in Patients with Breast Cancer: A Comparative Study with Ki67 and Phosphohistone H3. Journal of Surgical Oncology, 118, 557-567. https://doi.org/10.1002/jso.25152

[4] Zhang, C. and Qian, J. (2018) Research Progress on the Correlation between Ki67 and Tumor. Journal of Qiqihar Medical University, 39, 2536-2538.

[5] Sang, D., Wang, J. and Xu, B. (2015) Relationship between Proliferating Cell Nuclear Antigen Ki67 and Curative Effect of Neoadjuvant Chemotherapy for Breast Cancer. Chinese Journal of Breast Disease (Electronic Edition), 9, 50-53.

[6] Pathmanathan, N. and Balleine, R.L. (2013) Ki67 and Proliferation in Breast Cancer. Journal of Clinical Pathology, 66, 512-516.

[7] Chen, Q. and Wu, K. (2015) Research Progress on the Clinical Value of Ki-67 Breast Cancer and Its Critical Value Definition. Chinese Journal of Surgery, 53, 634-637.

[8] Qian, X., Cai, J., Zhao, J., et al. (2019) Expression and Significance of EBER, Ki67 
and BCL2 in Nasal NK/T Lymphoma. Journal of Nantong University (Medical Sciences), 39, 523-525.

[9] He, H., Chai, X., Xu, W., et al. (2020) Relationship between PD-L1, Ki67 and Clinicopathological Features and Prognosis of Patients with Non-Small Cell Lung Cancer. China Journal of Modern Medicine, 30, 39-44.

[10] Zhang, Y., Li, Q., Li, H., et al. (2019) Meta Analysis of the Prognostic Significance of Ki67 Expression Ratio in Breast Cancer Patients. Journal of Modern Oncology, 30, 1890-1894.

[11] Zheng, C., Wang, Y. and Lu, W. (2021) Diagnostic Value of Serum HE4 and Ki67 Levels in Patients with Ovarian Cancer and Their Relationship with Clinicopathological Features and Prognosis. Chinese Journal of Human Sexuality, 25, 45-49.

[12] Cao, Q. (2021) Expression of Serum VEGF, PD-L1 and Ki67 in Patients with Cervical Cancer and Its Prognostic Value. Chinese Journal of Public Health Engineering, 25, 256-258.

[13] Huang, T., Liu, J., Zhang, J., et al. (2019) The Role of Ki67 in Predicting the Prognosis of Prostate Cancer. National Journal of Andrology, 25, 1088-1092.

[14] World Health Organization (WHO) (2020) Global Health Estimates.

[15] Zuo, T., Zheng, R., Zeng, H., et al. (2017) Epidemiological Status of Gastric Cancer in China. Chinese Journal of Clinical Oncology, 44, 52-58.

[16] Xue, W., Fan, X. and Meng, G. (2014) Expert Consensus on the Selection of Immunohistochemical Markers Related to Gastric Cancer. Chinese Journal of Clinical and Experimental Pathology, 30, 951-953.

[17] Qiu, H. and Zhou, Z. (2018) Updates and Interpretation on NCCN Clinical Practice Guidelines for Gastric Cancer 2017 Version 5. Chinese Journal of Gastrointestinal Surgery, 21, 160-164.

[18] Wang, Y. (2021) Positive Expression of Ki67, EGFR and p53 in Gastric Cancer and Its Clinical Significance. Clinical Medicine \& Engineering, 28, 595-596.

[19] Zhao, S., Chen, Y., Li, H., et al. (2020) Correlation between Transcription Factor Foxq1 and Proliferation Index Ki67 in Advanced Gastric Cancer. Medical Journal of Communications, 34, 349-353.

[20] Yao, Q., Wang, J., Li, H., et al. (2021) Expression of NEDD9, CD44 and Ki67 in Gastric Cancer and Their Relationship with Clinicopathological Features. Journal of Modern Oncology, 29, 2863-2867.

[21] Chen, T., Chen, Y., Wu, J., et al. (2020) Expression of FOXP1 and Ki67 in Advanced Gastric Cancer and Their Effect on Prognosis. Journal of Nanjing Medical University (Natural Sciences), 40, 1181-1185, 1240.

[22] Jiang, S., Sun, D. and Xin, Y. (2019) Expression of WISP1 and Ki67 in Gastric Cancer and their Clinicopathological Significance. Journal of Modern Oncology, 27, 256-261.

[23] Zhou, Y., Chen, X. and Ding, H. (2016) Expression of TRAP1, Ki67 and AFP in Patients with Gastric Cancer and Their Combined Value in Predicting Liver Metastasis of Gastric Cancer. The Practical Journal of Cancer, 31, 1412-1413.

[24] Hua, Y. (2015) Correlation between Ki67 Expression and Chemosensitivity of Fluorouracil in Gastric Cancer. In: Shi, Y.K., Ed., the 9 th Chinese Symposium on Medical Oncology, CSMO, the 4th Annual Meeting of Chinese Society for Clinical Oncologists, CACO, Proceedings of 2015 Academic Annual Meeting of Tumor Clinical Chemotherapy Professional Committee of China Anti Cancer Association, Peking Union Medical College Press, Beijing, 770-771. 
[25] Cao, M. and Chen, W. (2021) Interpretation of GLOBOCAN 2020 Global Cancer Statistics. Chinese Journal of the Frontiers of Medical Science (Electronic Version), 13, 63-69.

[26] Hoshino, N., Hasegawa, S., Hida, K., et al. (2016) Nomogram for Predicting Recurrence in Stage II Colorectal Cancer. Acta Oncologica, 55, 1414-1417. https://doi.org/10.1080/0284186X.2016.1223881

[27] de Sousa, W.A.T., Rodrigues, L.V., da Silva, R.G., et al. (2012) Immunohistochemical Evaluation of p53 and Ki-67 Proteins in Colorectal Adenomas. Arquivos de Gastroenterologia, 49, 35-40. https://doi.org/10.1590/S0004-28032012000100007

[28] Li, H., Zheng, Y., Cheng, H., et al. (2021) Expression of Tumor Markers and Ki67 in Colorectal Cancer and Analysis of Prognostic Factors. Journal of Abdominal Surgery, 34, 110-114.

[29] Wang, X., Jin, L., Zhang, Y., et al. (2019) Expression of rpn11 and Ki67 in Colorectal Cancer and Their Clinicopathological Significance. Chinese Journal of Histochemistry and Cytochemistry, 28, 247-251.

[30] Tong, G., Zhang, G., Liu, J., et al. (2020) Cutoff of 25\% for Ki67 Expression Is a Good Classification Tool for Prognosis in Colorectal Cancer in the AJCC-8 Stratification. Oncology Reports, 43, 1187-1198. https://doi.org/10.3892/or.2020.7511

[31] Wei, X., Bu, J. and Mo, X. (2018) The Prognostic Significance of FBXO2 Expression in Colorectal Cancer. International Journal of Clinical and Experimental Pathology, 11, 5054-5062.

[32] Zhao, W., Lv, L., Qi, X., et al. (2016) Correlation between the Expression of CD147 and Ki67 in Hepatocellular Carcinoma and Its Clinical Significance. Military Medical Journal of South China, 30, 700-704.

[33] Chen, W. (2020) Diagnostic Value of Ki-67 and Ezrin Protein in Pathology of Primary Liver Cancer. Jilin Medical Journal, 41, 294-295.

[34] Zhang, X., Wu, Z.X., Peng, Y.H., et al. (2021) Correlationship between Ki67, VEGF, p53 and Hepatocellular Carcinoma Recurrence in Liver Transplant Patients. BioMed Research International, 2021, Article ID: 6651397. https://doi.org/10.1155/2021/6651397

[35] Guo, F., Yang, J., Xiong, S., et al. (2018) Expression of Drug Resistance Gene Protein and Ki67 in Primary Liver Cancer and Its Prognostic Value. Chinese Journal of Hepatic Surgery (Electronic Edition), 7, 77-81.

[36] Jia, L., Li, H., Bai, H., et al. (2020) The Expression of CD34 and Ki67 Predicts the Risk of Postoperative Recurrence of Hepatocellular Carcinoma. Chinese Hepatology, 25, 197-199.

[37] Chen, W.Q., Zheng, R.S., Zeng, H.M., et al. (2016) The Incidence and Mortality of major Cancers in China, 2012. Chinese Journal of Cancer, 35, Article No. 73. https://doi.org/10.1186/s40880-016-0137-8

[38] Siegel, R.L., Miller, K.D. and Jemal, A. (2019) Cancer Statistics, 2019. CA: A Cancer Journal for Clinicians, 69, 7-34. https://doi.org/10.3322/caac.21551

[39] Wang, H., Zhou, Y., Liu, Q., et al. (2018) Prognostic Value of SOX2, Cyclin D1, P53, Ki-67 in Patients with Esophageal Squamous Cell Carcinoma. OncoTargets and Therapy, 11, 5171-5181. https://doi.org/10.2147/OTT.S160066

[40] He, X., Ma, R., Jia, W., et al. (2020) Expression and Clinical Significance of S100A8 and Ki67 Proteins in Esophageal Carcinoma. Labeled Immunoassays and Clinical Medicine, 27, 989-992, 995.

[41] Qi, X., Qiu, Y., Chen, D., et al. (2019) Expression of Ki-67 and VEGF in Esophageal 
Carcinoma and Their Relationship with Radiotherapy Prognosis. Chinese Journal of Clinical Rational Drug Use, 12, 44-46, 48.

[42] Zhao, L., Chu, X., Li, S., et al. (2021) Expression and Significance of Ki-67 in Basal Cell Like Squamous Cell Carcinoma of Esophagus. People's Military Surgeon, 64, 446-449.

[43] Jacobsen, F., Kohsar, J., Gebauer, F., et al. (2020) Loss of p16 and High Ki67 Labeling Index Is Associated with Poor Outcome in Esophageal Carcinoma. Oncotarget, 11, 1007-1016. https://doi.org/10.18632/oncotarget.27507

[44] Deng, H.Y., Chen, Z.H., Wang, Z.Q., et al. (2017) High Expression of Ki-67 Is an Independent Favorable Prognostic Factor for Esophageal Small Cell Carcinoma. Oncotarget, 8, 55298-55307. https://doi.org/10.18632/oncotarget.19426 